\title{
COMMENTS ON THE COURSE OF SOLAR ACTIVITY DURING THE DECLINING PHASE OF SOLAR CYCLE 20 (1970-74)
}

\author{
H. W. DODSON and E. R. HEDEMAN \\ McMath-Hulbert Observatory of The University of Michigan, \\ 895 Lake Angelus Road, Pontiac, Mich., 48055, U.S.A.
}

(Received 7 March, 1975)

\begin{abstract}
In the declining phase of solar cycle 20 (1970-74) three pulses of activity occurred and resulted in two well defined 'stillstands' in the smoothed means of sunspot, $2800 \mathrm{MHz}$, and calcium plage data. Marked diminutions in spot and $2800 \mathrm{MHz}$ flux took place in 1970 and 1971, respectively, and were accompanied by concomitant decreases in flare-occurrence. Studies of the latitude distribution of spots and flares show the extent of the dominance of the northern hemisphere in cycle 20 and the marked phase shift between northern and southern hemispheres. In the years studied, the longitudes of centers of activity clustered in identifiable zones or hemispheres for relatively long intervals of time. From mid-1973 to mid-1974 the Sun had a relatively inactive hemisphere centered on $\sim 0^{\circ}$ longitude. The relationship of certain well defined 'coronal holes' to this inactive hemisphere of the chromosphere is noted. The first two spot groups of the new cycle formed in November 1974 and January 1975 in the longitude zone associated with relatively high levels of old cycle activity, a repetition of the pattern observed in 1963-64.
\end{abstract}

\section{Introduction}

After a long-enduring maximum extending from 1968 through late 1970 solar activity, as measured by sunspot numbers, $2800 \mathrm{MHz}$ flux, and calcium plages declined irregularly. Figure 1 shows the course of activity through 1974 as indicated by these parameters and gives a plot of mean values for each Carrington rotation, as well as the 13-rotation running means of the respective quantities.

\section{Pulses in Activity}

According to our evaluation of the data, since 1970 there have been three apparent pulses in the level of activity as indicated by the individual rotational values. The first of these increases lasted from about July 1971 to the autumn of 1972 and resulted in a conspicuous 'stillstand' on the declining branch of each of the activity curves (smoothed values). This pulse in activity was a result primarily of sunspot activity in the southern hemisphere. The 'stillstand' in the plot of 13-month running means of sunspot numbers was similar in magnitude to the especially well defined 'stillstands' of cycles 10 and 18 (see Figure 2). The second pulse in activity as shown by the rotational values was small and took place during the first three quarters of 1973. It included an interval in March and April with numerous large, bright plages and magnetically complex spots, and a time in August and September with very large numbers of small spot groups. The third pulse began in April 1974 and was of sufficient magnitude to introduce a second well defined 'stillstand' on the declining branch of activity curves for cycle 20 (see Figures 1 and 2). Two well defined 'still- 
MEAN ZURICH SUNSPOT NUMBER

CYCLE 20

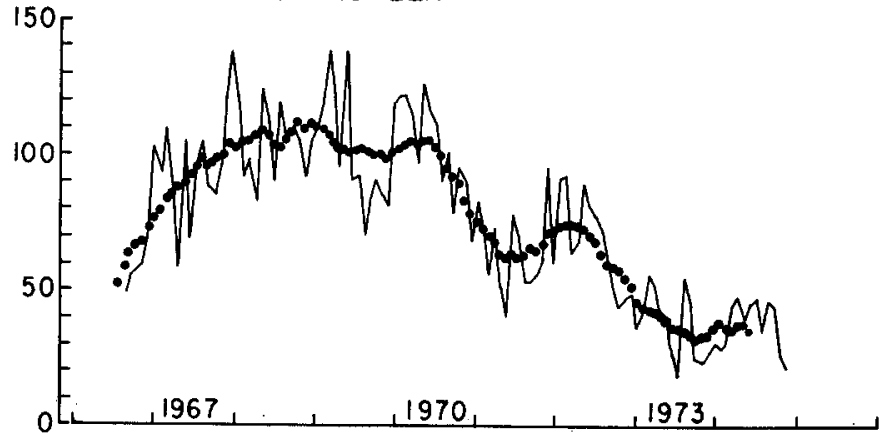

MEAN $2800 M_{H Z}$ FLUX

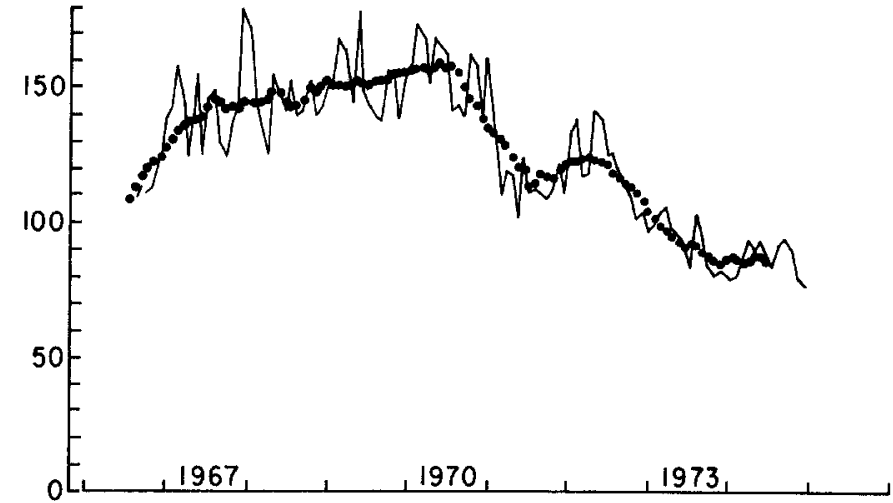

MEAN A X I (Calcium Plages)

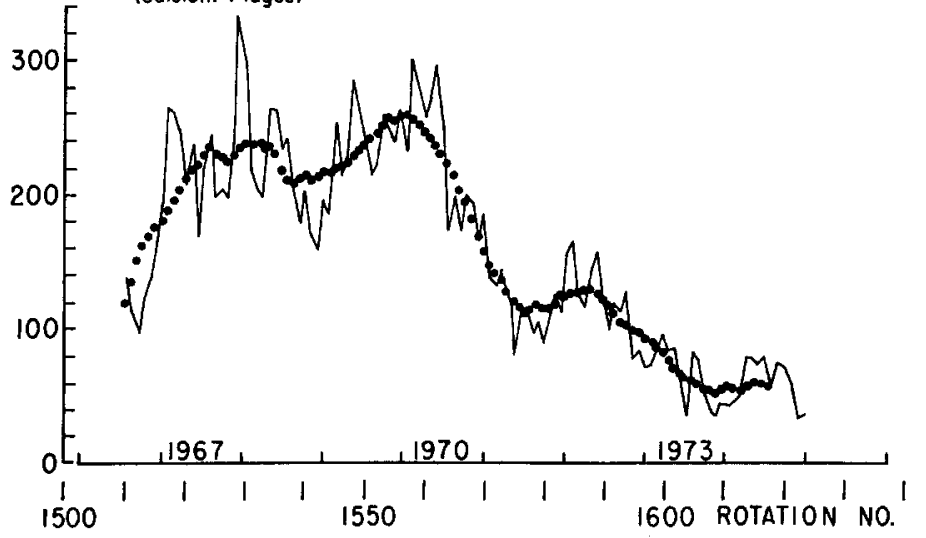

Fig. 1. Plot of mean values for each Carrington rotation and smoothed 13-rotation running means for Zürich sunspot numbers, $2800 \mathrm{MHz}$ flux (Ottawa), and $\Sigma$ area $\times$ excess intensity for calcium plages (McMath-Hulbert Observatory). 
SMOOTHED MONTHLY MEAN
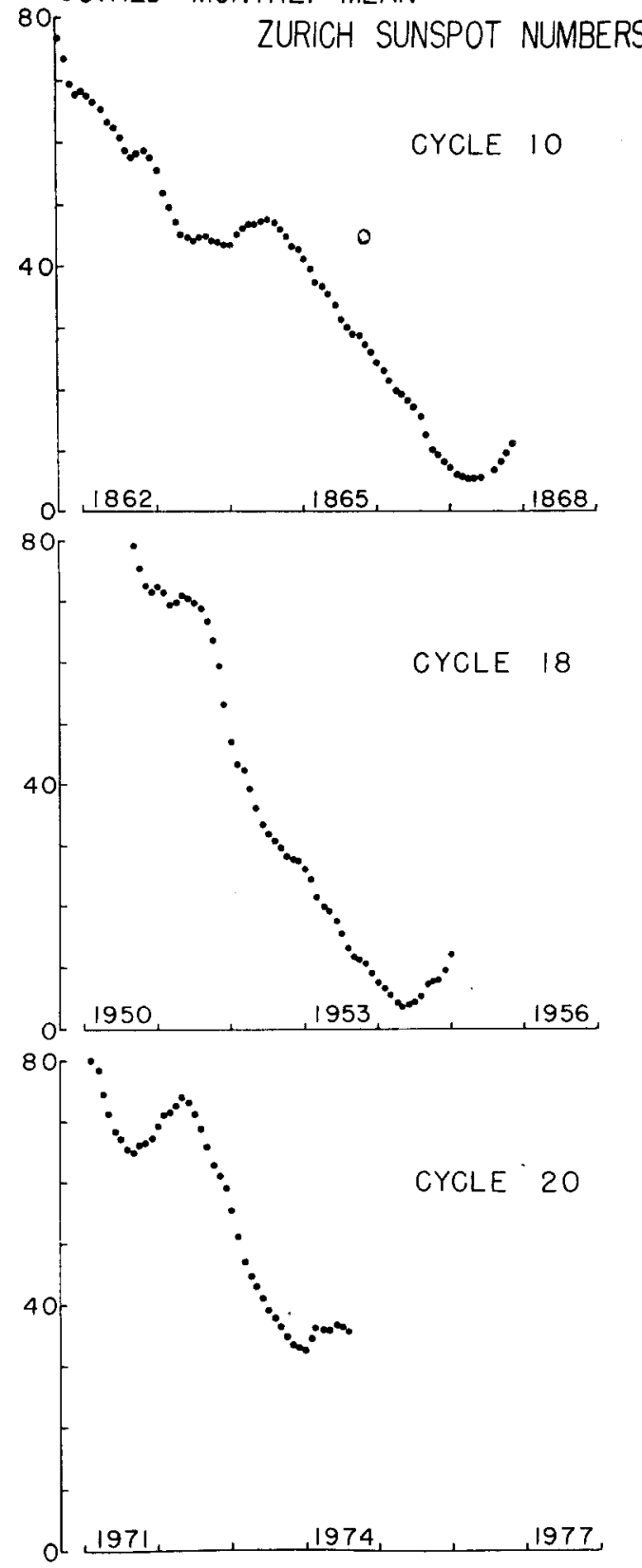

Fig. 2. Smoothed monthly means of Zürich sunspot numbers for portions of solar cycles 10, 18, and 20. 
stands' during the later years of a cycle seem a little unusual, although a number of other cycles have shown multiple undulations in the smoothed sunspot numbers as they fell from maximum to minimum values. (See cycles 2, 11, 15; Waldmeier, 1961.)

\section{Marked Decreases in Activity}

Mid 1970 to mid 1971 was an interval of rapidly decreasing activity as measured by rotational mean sunspot numbers (see Figure 1). However, a similarly sustained decline in rotational means for $2800 \mathrm{MHz}$ did not begin until seven months later in February 1971. It is not surprising to find that these times of marked decline in sunspot numbers and $2800 \mathrm{MHz}$ flux are reflected in concomitant diminutions in the number of flares of importance $>1$, first in the southern hemisphere in mid 1970 and in the northern hemisphere at the start of 1971 (see Figure 3a). It was in these months when solar circumstances were first beginning to approach those of solar minimum, and exactly at the time of the first sustained interval of especially low flare production in both the northern and southern hemisphere (January-July 1971) that the first well defined sequence of 27-day recurrent geomagnetic storms took place in solar cycle 20. It is interesting that during 1971, 1972, and the first half of 1973, flare production in general was relatively uniform with the flare-rich months of August 1971 and August and October 1972 showing as exceptional circumstances. In July 1973, flare frequency dropped to a new and lower level and remained low for nine months. It can be noted that the Skylab missions took place primarily during this interval in which flares of importance $>1$ were especially infrequent. In April 1974, when the aforementioned third pulse of activity began, flare frequency increased and remained relatively high through October 1974. The flare events in July 1974 were especially noteworthy (see Figure 3a).

\section{Latitude Distribution}

Attempts can be made to describe the general location of centers of activity on the Sun during the past four years. It is well known that throughout cycle 20, spot activity has been greater in the northern than in the southern hemisphere. Figure $3 \mathrm{~b}$ demonstrates the extent of this imbalance through a plot of the monthly mean values of daily sunspot areas in the northern and southern hemispheres respectively. For 1965-1973 the areas are those published by the Catania Observatory, and for 1974 the values were taken from the Solar Data Bulletin, U.S.S.R. In addition, Figure $3 b$ shows that there has been a phase shift between the two hemispheres. For the first 16 months of cycle 20 , new cycle spots were confined to the northern hemisphere. In the declining years of the cycle, monthly mean sunspot areas in the northern hemisphere fell to relatively low levels as early as 1971. A similar decline in southern sunspot areas did not take place until 1973. That solar activity has been greater in the southern than in the northern hemisphere in the later years of cycle 20 also can be seen in the plot of flares in Figure 3a. There is a general preponderance of flares of importance $>1$ in the southern hemisphere starting in August 1971. 

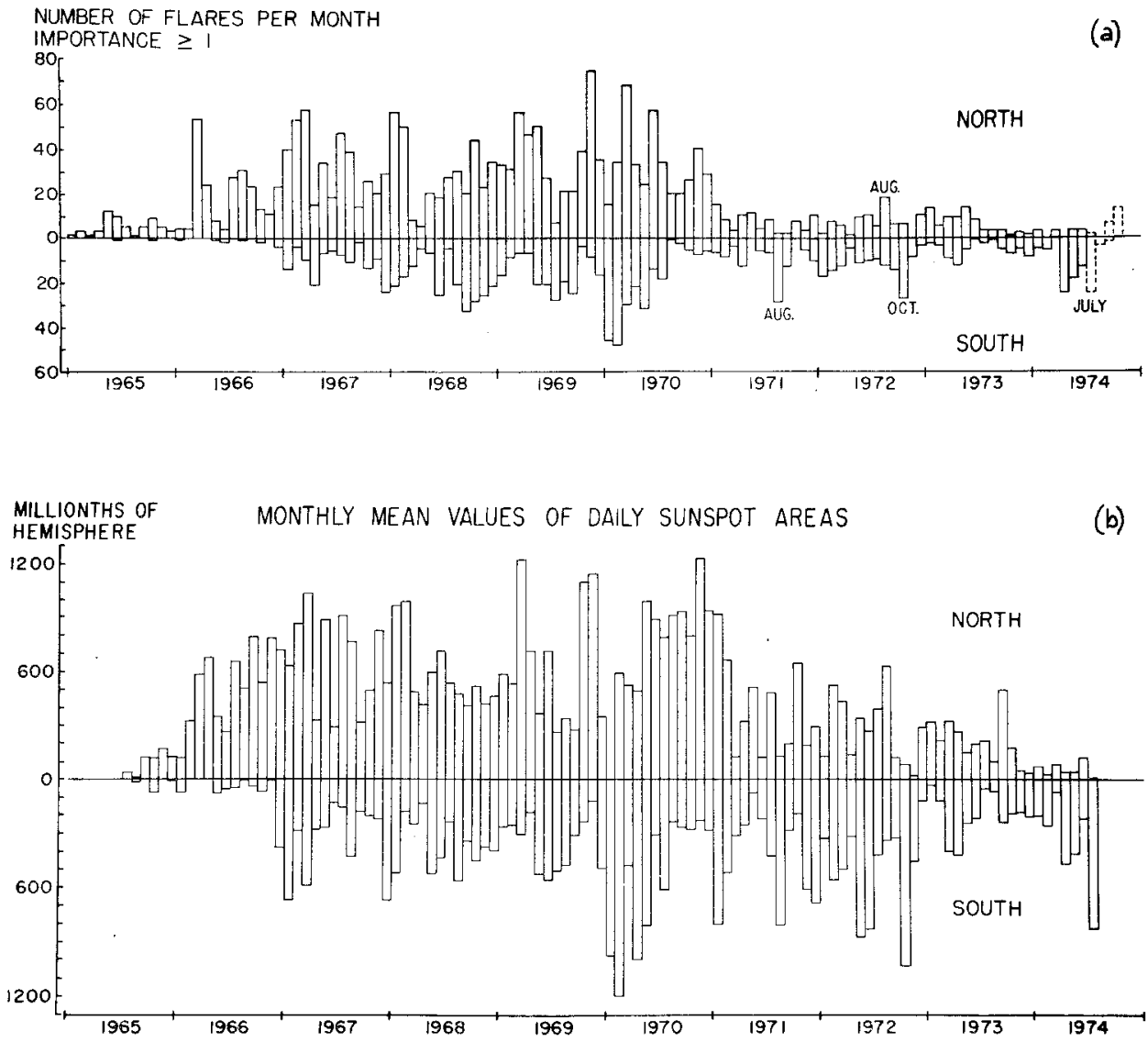

Fig. 3. (a) Number of fiares per month of importance $\geqslant 1$ for the years 1965-1974, according to location in northern or southern hemisphere. (Data from $Q B S A$, but reevaluated for 1965-1967.) (b) Monthly mean values of daily sunspot areas by northern and southern hemispheres, 1965-1974. (Data from Catania Observatory 1965-1973 and from Solar Data Bulletin, U.S.S.R. for 1974.)

\section{Longitude Distribution}

The longitudes of centers of activity are much more difficult to describe than the latitudes. In Figure 4, a plot, by Carrington longitude and rotation, has been made of all centers of activity with large or bright plages and with spots. The 'antecedents and descendents' of each such region are shown. The size of the circles reflects the size of the plage. Heavy dark circles identify flare-rich regions and give an indication of the flare frequency. Black squares outline the centers of activity associated with the principal energetic particle events. We are aware that Carrington longitudes represent a frame of reference for only one rotation period ( $\sim 27.3$ days $)$ and that differential rotation and/or systematic patterns of region formation and growth suggest other possible organizations of the data. We shall try, nevertheless, to describe the observed activity in terms of Carrington longitudes. 


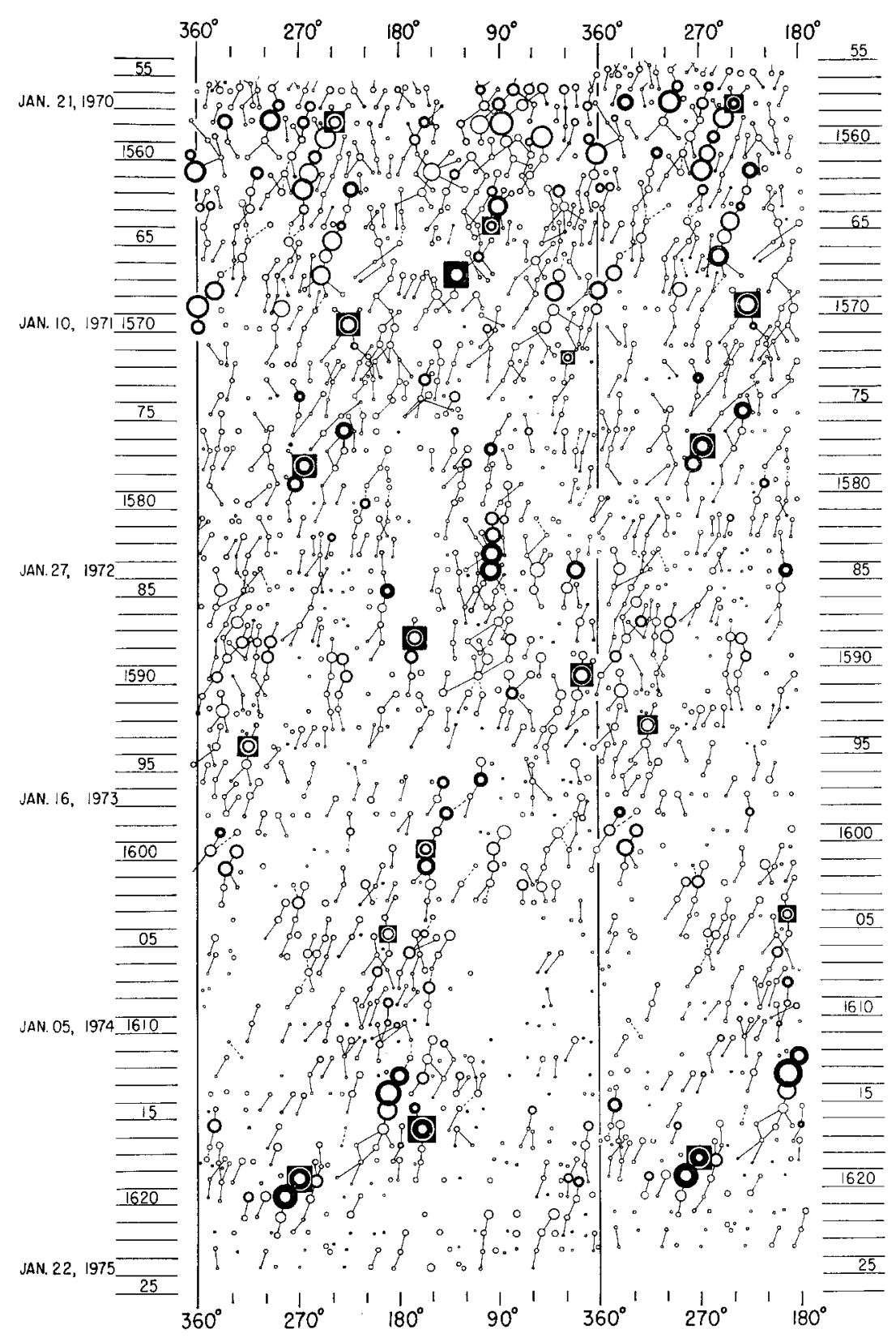

Fig. 4. Plot by Carrington rotation number and longitude of all plages with area 1000 millionths of the solar hemisphere and/or intensity 3, 1970-1974. The 'antecedents' and 'descendents' of each region also are included. The size of the circle is an indication of the area of the plage. Heavy dark rings indicate especially flare-rich plages. A dark square outline identifies the regions associated with proton emission. Time runs from left to right and from top to bottom. Data are repeated for $1 \frac{1}{2}$ rotations. 


\subsection{ACTIVITY IN 1970-1971}

In 1972, the authors published a similar plot of centers of activity for the years 1969 and 1970, and wrote as follows: "The spatial distribution of solar activity during the two years, 1969 and 1970, has emphasized anew the persistence of activity in preferred longitudes on approximately opposite sides of the Sun. During these two years, the solar surface can be said to have exhibited four well-defined activity sectors, two strong and two weak. The zones in 1969 and 1970 with high activity were centered at Carrington longitudes of $\sim 80^{\circ}$ and $\sim 260^{\circ}$. The zones of weak activity were centered at $\sim 0^{\circ}$ and $\sim 180^{\circ} . "$ The above description, of course, refers also to the data for 1970 as plotted in the top portion of Figure 4. In 1971, the same pattern of relatively active and quiet longitudes can be discerned, although in this year, the zones without major centers of activity at $\sim 0^{\circ}$ and $\sim 180^{\circ}$, are better defined than the active longitudes.

\subsection{Activity IN 1972-1974}

In 1972 a conspicuous change took place in the location of activity on the solar surface. Significant centers of activity developed in the two longitude zones, $\sim 0^{\circ}$ and $\sim 180^{\circ}$ that for three years had been relatively deficient in such phenomena. In rotation 1584, in the first of these zones, flare-rich McMath plage 11748, (N10 $0^{\circ}$ longitude $21^{\circ}$ ), crossed the central meridian on February 22, 1972. It was the site of two $\beta \gamma$ spots and had 32 flares and subflares which were associated with sudden ionospheric disturbances (SID). This was the largest number of SID's associated with any center of activity in 1972. Later in 1972, the well known flare- and proton-producing region with CMP August 4, 1972 (rotation 1590) occurred in a very similar location (N13,
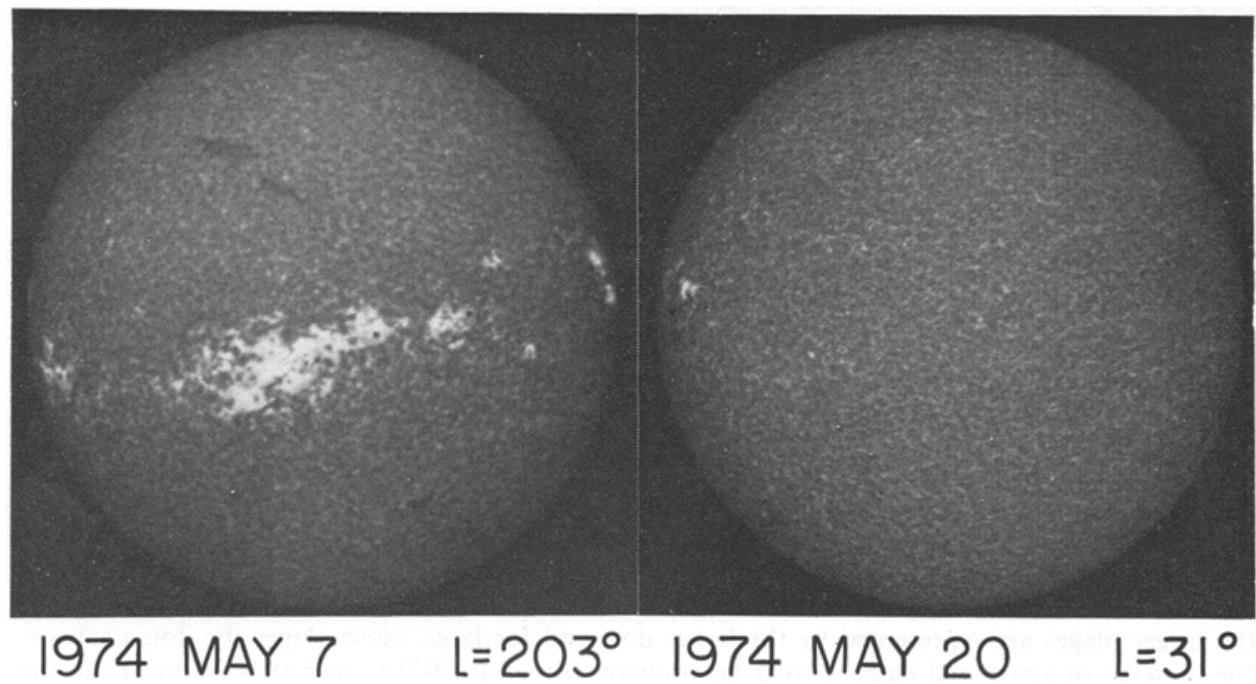

Fig. 5. Calcium spectroheliograms showing the active and inactive hemispheres in May 1974. 
longitude $13^{\circ}$ ) and was followed in October 1972 (rotation 1594) by a major, southern center of activity $\left(\mathrm{S} 12^{\circ}\right.$, longitude $315^{\circ}$ ) also in the same general longitude zone. Above average activity continued in this longitude zone centered on $\sim 0^{\circ}$ through rotation 1601 .

The increase in activity in this longitude zone lasted from approximately February 1972 through May 1973. In subsequent rotations activity in the entire hemisphere centered on $\sim 0^{\circ}$ diminished greatly, and this hemisphere of quiet continued until mid 1974. For almost a year (mid 1973-mid 1974), the difference between the active and inactive hemispheres constituted one of the most conspicuous aspects of the Sun.
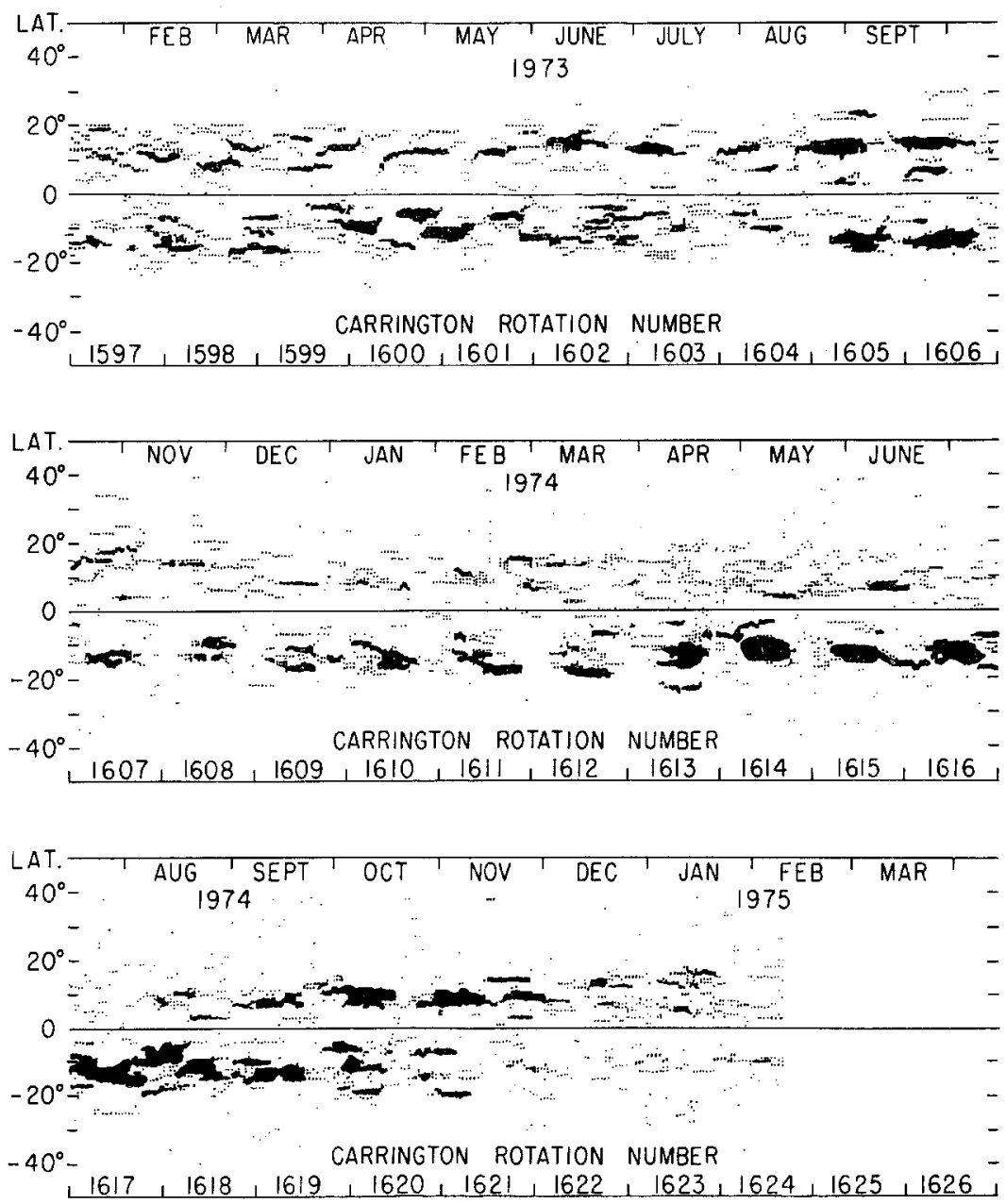

Fig. 6. A plot vs time of the latitude of each plage observed each day (January 1973-February 1975). The larger plages are represented by the larger dots and for large, close plages the dots coalesce. The presence of active and inactive solar hemispheres from mid-1973 to mid-1974 is obvious in the data. Observations from the McMath-Hulbert Observatory have been supplemented by reduction of spectroheliograms from Manila for days of no observation at the McMath-Hulbert Observatory. 
Figure 5 shows an example of the difference in the two hemispheres as recorded on calcium spectroheliograms. A daily plot of each plage by latitude likewise shows the extent of this sustained pattern in basic chromospheric activity (see Figure 6).

Solar quiet in 1971 that was centered on longitude $\sim 180^{\circ}$ was broken in rotation 1588 (May 1972) with the development of proton-producing, flare-rich McMath plage $11895, \mathrm{~N} 09^{\circ}$, longitude $167^{\circ}$ (CMP May 30, 1972). After another interval of relative quiet, activity in this zone resumed in rotation 1600 (April 1973) and continued through at least rotation 1616 (July 1974) with the passage of the significant flare- and proton-producing center of activity McMath plage $13043, \mathrm{~S} 14^{\circ}$ longitude $155^{\circ}$ (CMP July 4, 1974).

In rotations 1619 and 1620 (September and October, 1974), Figure 5 shows the occurrence of a flare-rich region in longitude $\sim 270^{\circ}\left(\mathrm{S} 08^{\circ}\right)$. The location of this center of activity emphasizes the difficulties and ambiguities inherent in efforts to recognize large-scale zones of activity on the Sun. Does this region form a part of the activity zone here described as centered on $180^{\circ}$ ? It lies on the preceding boundary of the zone. Perhaps more importantly, it occurred in the track of the apparent westward drift of the 'family' of centers of activity associated with this zone.

\subsection{General comments}

In spite of the uncertainties and ambiguities in efforts to organize chromospheric activity, it seems clear from Figure 4 that, during the past four years, large plages and flare-rich centers of activity have clustered in identifiable zones or hemispheres for relatively long intervals of time. Other zones or hemispheres have been exceptionally quiet. At the present time, relationships between these gross chromospheric patterns and coronal or interplanetary phenomena appear to be complex. It is interesting to note, however, that three of the apparently best defined 'coronal holes' as observed by OSO-7 and/or Skylab equipment, appear to have been located near the middle of the hemisphere that was so quiet in the chromosphere from mid 1973 to mid 1974 (centered on $\sim 0^{\circ}$ ), and at longitudes very similar to that of the prior and very great center of activity in August 1972. We refer to the following coronal holes:

CMP $\sim$ January 16, 1973, longitude $\sim 9^{\circ} \quad$ (Neupert and Pizzo, 1974)

CMP $\sim$ May 31, 1973, longitude $\sim 22^{\circ} \quad$ (Sky and Telescope, July 1974)

CMP $\sim$ August 20,1973 , longitude $\sim 31^{\circ} \quad$ (Sky and Telescope, July 1974)

At the present time, a clear understanding of the relationships and evolutionary development of the various aspects of solar activity and solar quiet seems far in the future.

\section{New Cycle Activity}

Finally, it can be reported that as of January 1975, two spots of the new cycle have been observed; one in November 1974 in the north in longitude $\sim 250^{\circ}$, latitude $\mathrm{N} 37^{\circ}$, and a second in January 1975 , in the south at $\sim 165^{\circ}$ longitude and latitude $\mathrm{S} 26^{\circ}$. These new cycle spots developed within the hemisphere centered on $\sim 180^{\circ}$ and in the zone associated at that time with the higher levels of old cycle activity. 
These new cycle spots present a situation similar in some respects to the outbreak of new cycle regions eleven years ago. At that time we wrote (Dodson and Hedeman, 1969) "In 1963-65 new cycle regions developed in close juxtaposition to major oldcycle regions and in the same longitudes that were and had been the favored zones for old cycle activity." Although the patterns of activity on the Sun were markedly different in 1963 and in 1974 there apparently was some element of similarity in the phenomena. It will be interesting to compare further the decline of cycle 20 and the onset of cycle 21 as these circumstances become known in 1975 and 1976.

\section{Acknowledgements}

The authors acknowledge with gratitude the support of the National Aeronautics and Space Administration through contract NGL-23-005-275 in the preparation of this study. We also want to thank especially our colleagues, Mr Clifford Bennett and Mr William Marquette, for their special help in the preparation of the diagrams used in this paper.

\section{References}

Dodson, H. W. and Hedeman, E. R.: 1969, in Annals of the IQSY, M.I.T. Press, Cambridge, Mass., $4,3$.

Dodson, H. W. and Hedeman, E. R.: 1972, Progress in Astronautics and Aeronautics 30, 19.

Neupert, W. M. and Pizzo, V.: 1974, J. Geophys. Res. 79, 3701.

Sky and Telescope: 1974, 48, 11.

Waldmeier, M.: 1961, The Sunspot-Activity in the Years 1610-1960, Zürich Schulthess and Co. 